\title{
An Agent-Based Model of Tourist Movements in New Zealand: Implications for Spatial Yield
}

\author{
$\underline{\text { C. Doscher }}^{\text {a }}$, K. Moore ${ }^{\text {a }}$, C. Smallman ${ }^{\text {b }}$, J. Wilson ${ }^{\text {a }}$, and D. Simmons ${ }^{\text {a }}$ \\ ${ }^{a}$ Faculty of Environment, Society and Design, Lincoln University, Lincoln, New Zealand \\ Email: crile.doscher@lincoln.ac.nz \\ ${ }^{b}$ School of Management, University of Western Sydney, Sydney, Australia
}

\begin{abstract}
Tourism is New Zealand's fourth largest industry, providing jobs for thousands of New Zealanders and significant foreign capital for the nation's economy. Of concern to ministry and industry decision makers is the "spatial yield" of these tourists which takes into account the spatial and temporal contributions of their movements in terms of economic, cultural and environmental impacts. We have developed an agent-based model of tourism movements to simulate these impacts and to allow for the evaluation of different scenarios (such as increases in petrol prices or variations in currency exchange rates) on the behaviours of those tourists. In order to develop realistic and grounded heuristics for the model, interview protocols were developed in order to identify the key drivers in tourists' decision making process.
\end{abstract}

140 interviews were conducted in five locations in the Canterbury region of New Zealand's South island. Demographics collected included gender, age, nationality, country of residence, travel group details, type of transport, and length of stay were also collected. Based on the experiences of the interviewers and a review of the results, a second interview protocol was developed which focused only on what tourists had done on the previous day or immediately before the interview. Key results from the interviews allowed us to derive several factors: Trip Type (purpose of the visit) which correlated well with itinerary types, accommodation levels, and transport types. When nationality is included, this also correlated well with total budgets. These results were compared against data collected in the annual International Visitor Survey. Interviews also revealed that the decision making process changed over the course of a trip, with decisions like accommodation booking being locked in during the first third of a trip and becoming less constrained as the trip continued. We propose a "cascading" model of decision-making where initial decisions (often made prior to arrival in New Zealand) influence the flow of later, in-country decisions.

The model is being developed using Repast Simphony. The primary agents are travel groups portioned by nationality, trip type, itinerary type, accommodation level and type of transport. The preliminary model will simulate the period of 1 November 2008 to 28 February 2009 with inputs based on data from the IVS, allowing for simulations to be validated against these data. Once validated, the model will allow us to simulate different scenarios and their impact on spatial yield. In addition, the impacts of weather events and natural disasters (e.g., earthquakes and volcanic eruptions) on tourist movements. This paper presents the outlines the development of the model as well as a methodology for translating qualitative interview data into decision-making heuristics.

Keywords: $\quad$ Agent based modeling, simulation, interviews, tourism 


\section{INTRODUCTION}

As New Zealand's fourth largest industry, tourism plays a crucial role in the country's economy. Aside from providing jobs for thousands of New Zealanders, the foreign currency brought in by international tourists provides significant foreign exchange benefits and supports large portions of the rural economy. In the year ending in March, 2010, tourism contributed NZ\$6.5 billion to the economy, or roughly $3.8 \%$ to the country's total GDP (Ministry of Economic Development, 2011). An important characteristic of tourism is its spatial and temporal nature. International tourists may spend anywhere between two days and several months touring the country, and in so doing, spread their economic, environmental and social impacts over a range of spatial extents, an impact we refer to as their "spatial yield". To gain a better sense of the effects of spatial yield as well as to provide a model to aid decision makers to optimise the benefits from tourism, we have developed an agent-based model of tourism movements around New Zealand which is grounded in decisionmaking data collected from tourists via semi-structured interviews. Analysis of these interviews provided insights into the factors that affect tourists' decision-making processes and allowed us to develop heuristics for modeling purposes. This paper will outline the model and its development as well as focusing on the methodology through which movement heuristics were developed based on interview data collected with international tourists.

\section{DATA COLLECTION}

Semi-structured interviews were carried out by intercepting tourists in-situ resulting in 140 interviews at five separate locations in the Canterbury region of New Zealand's South Island. The primary focus of these interviews was to identify the key drivers of the decision-making process by probing how they came to decisions for such choices as their destinations, their overnight accommodation and the activities they took part in. Demographics including gender, age, nationality, country of residence, travel group details, type of transport, and length of stay were also collected. The sites chosen for the interviews represented different destination sites: a 'gateway' or entrance point into New Zealand (Christchurch), a 'terminal' or diversion from a main road site (Akaora and Hanmer Springs), and a 'through-route' site located on a main throughroute (Kaikoura and Tekapo). This allowed us to sample tourists at various points in their trip as well as at different destination types. Based on the experiences of the interviewers and a review of the results, a second interview protocol was developed which focused only on what tourists had done on the previous day or immediately before the interview.

The interview data were analysed both manually and electronically (using NVivo software). Lengths of stay ranged from six days to one year (Moore et al., 2009). An important variable that emerged from the data analysis was that tourists could be categorised by their "Trip Type" related to the purpose of the visit. The primary categories of Type of Trip are sightseeing, and visiting friends or relatives (VFR), holiday/family, working holiday and 'round the world' (RTW). The category a tourist group was classified into tended to correlate well with the style of travel, itineraries, transport and accommodation choices. Another important result suggested that, in general, the first third of their trip was more planned while the middle and final thirds were less planned and structured, indicating that tourists' decision-making evolved as they became more familiar with the ease of travel in the country. In addition, during the initial third of the trip, accommodation choices were more or less "locked in" and became more open ended as the trip progressed.

The interview analysis also suggested that there were four important dimensions of decision-making that could influence tourist behaviours: (In)Flexibility; timing/location; social composition; and stage of trip (as just discussed). (In)Flexibility relates to the perceived 'ease' of travel in New Zealand, the level of decision openness and the openness of tourists to advice and information. The dimension of timing/location calibrates when and where decisions were made and includes the number of decisions that may have been made before arriving and could be influenced by the perceived risks of not booking accommodation or activities in advance as well as the needs of the particular group members. 'Social composition' concerns the range of social influences upon the decisions made by tourists or groups of tourists. In particular, it represents the effect that travel group composition has on decisions made, such as the presence of young children, or the presence of either a New Zealander or a previous visitor in the group. It also includes, however, the effect of others beyond the group (e.g., other tourists, locals encountered and friends and relatives in New Zealand but who may not be travelling with the tourist or travel group. Additionally, the observation that the decision making process appears to change with length of time in the country, reflected in the stage of trip, allows us to breakdown trips into thirds, typified by different styles of decision making.

This analysis allows us to propose a 'cascade' model of decision-making where the Type of Trip a tourist group is on leads to a particular cascade of decision-making that is influenced by the remaining three basic dimensions of identified above (see figure 1). 


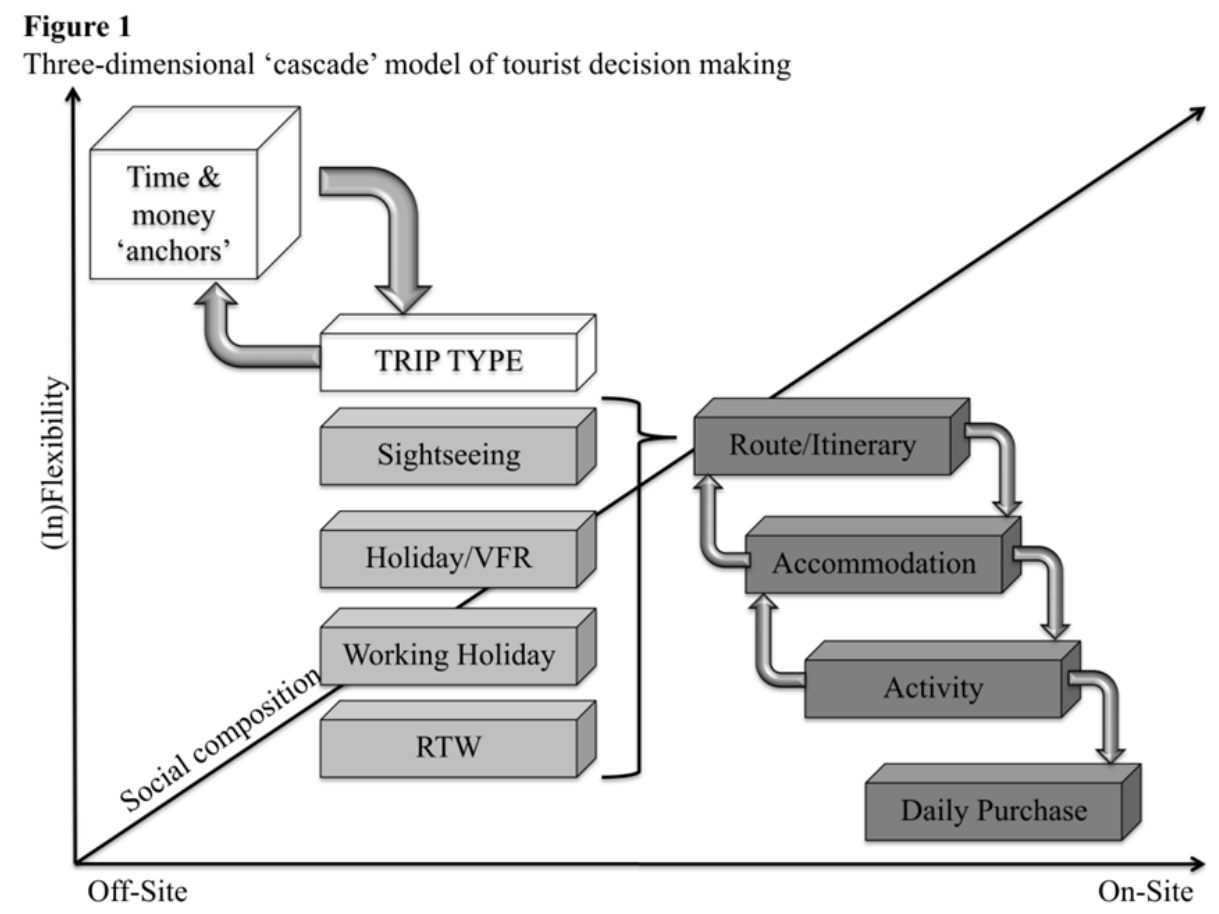

Available time and budget anchor or bookend the trip and, importantly, determine the Trip Type prior to arrival in New Zealand. Once decided, this provides a framework for the subsequent classification of agents in the model.

An example should help to illustrate how these dimensions interact to produce tourist decisions and behaviour. A travel group on a 'holiday/family' Type of Trip (e.g., often from Australia for a short duration) may well be relatively inflexible (i.e., high 'Inflexibility') about accommodation and itinerary choices because those decisions were made prior to travel and off-site (i.e., early/off-site 'timing/location') solely by the adults/parents (i.e., low/closed 'social composition'); activity decisions may be partly inflexible (i.e., moderate '(In)Flexibility' - e.g., a child in the family wishes to do the 'luge' at Rotorua but some other activities remain flexible) and have relatively high 'social composition' (e.g., other family members influence the decisions as may recommendations of locals or New Zealand friends/family); further, activity decisions may not be finalized until on-site (i.e., late/on-site 'timing/location') but channeled by prior information (and the 'luge' preference of a family member); daily purchases, including what and where to eat may be relatively flexible (i.e., moderate to high '(In)Flexibility' - though constrained, perhaps, by cost and dietary preferences and needs) and open to influence by locals (e.g., the recommendation of the owner of the motel where the group is staying); even during a relatively short stay some flexibility may be built into the middle part of the trip (e.g., have a day trip to either destination A or B at about mid-trip).

These dimensions - and their magnitude in relation to particular types of decisions (e.g., accommodation, activities, itinerary, etc. decisions) made, as they are, within particular Types of Trip - then interact with the decision making process to affect the kinds of heuristics that are 'chosen' to make - or simply emerge as the means of resolving - particular moment by moment decisions. That is, the heuristics that have been identified in the extensive literature on human decision-making are effectively 'sieved' through the matrix created by the dimensions identified from the data in the qualitative interviews in relation to particular types of decisions within the context of particular Types of Trip. In the case of the 'holiday/family' Trip Type, for example, relatively deliberative heuristics (e.g., elimination by aspects) may be used to come to activity decisions that have moderate flexibility, involve relatively high social composition (e.g., other family members) and are likely to be made in New Zealand but prior to arrival at particular sites. By contrast, daily purchases that are highly flexible, low in social composition (e.g., up to an individual) and made at the point of the behaviour (e.g., in a shop) may be determined by less deliberative heuristics (e.g., take the best immediately on offer within a set price range). Inspection of the IVS revealed that typical itineraries could be placed into one of three groups: loop (multiple nodes starting and stopping at the same node), triangle (three nodes starting and 
stopping at the same node) or stationary (staying primarily at the same node but making short trips out and back).

An important point, is that the nature of this dimensional matrix' will be specific, in the model, to each kind of decision for each kind of Type of Trip. In addition, it will be affected by the particular 'third' of the trip within which that decision is being made. The fieldwork was also designed so that the qualitatively derived 'agent categories' of Trip Type could be 'cashed out' in 'fuzzy sets' of variables (such as nationality, length of stay, repeat visitation, age, transport type, accommodation type, etc.) that have been used as the basis for standard data collection on international tourists in New Zealand (principally, the International Visitors Survey (IVS)). This survey is carried out annually by the Ministry for Economic Development in order to capture travel patterns and expenditure of international tourists and contains questions relating to places visited, expenditure, places stayed, accommodation and activities/attractions undertaken (Ministry of Economic Development, 2011).

Because of the spatial and temporal nature of tourists' activities, an agent-based model was developed as a way to simulate tourist flows as well as provide a tool to evaluate the effect of changes that may have an impact on the spatial yield of tourists, such as changes to the mix of tourists, variations in exchange rates, or even the effects of natural disaster (e.g. earthquakes, volcanic eruptions). The model is still in the early stages of development and the following sections outline the model structure and how the interview data were used to inform its development.

\section{MODEL DEVELOPMENT}

\subsection{ABM Platform}

Repast Simphony was chosen as the agent-based modeling platform based on its flexibility and capability of working with ESRI Shapefiles (North et al., 2006, ROAD, 2009). This Java-based platform is freely available open source software that is widely used for ABM applications. Days are simulated starting at 9.00 in the morning and running in one hour timesteps until 11.00 at night. On startup, the model builds up a simulated New Zealand environment (NZWorld) composed of a road network and nodes. The nodes represent any location where tourists undertake activities and roughly approximates to towns and cities, though some nodes will occur outside of these locales. Each node contains representations of activities, accommodation and restaurants which agents can query and interact with upon arrival at a node. The locations of nodes are read into the model as a point shapefile and additional data (accommodation, activities, restaurants and accompanying costs) are read in from a comma separated (CSV) file. Travel group arrivals and their attributes are also read in to the model from a CSV file. NZWorld also functions as a timekeeper of the simulation, breaking the simulated day up into three primary time slots for meals and activities. Once instantiated, agents check into a virtual hotel for their first day and are endowed with a total budget, a daily spending limit, a preferred level of accommodation and a list to hold activities already done. During the development phases, the groups itinerary is set based on the total number of days in their trip, their arrival and departure nodes and their itinerary type (e.g. loop, triangle, or stationary). As noted previously, later versions of the model will provide the travel groups more capability to choose their own destinations.

The model is broken into two modules to represent between-node and in-node decisions. Between node behaviours refer to decisions made in the process of travelling from one node to the next in the course of the trip. Since agents initially stay in their port of arrival, their first day is driven by in-node decisions that include eating at a particular time of day and choosing and doing activities. Agents can query a node to determine what activities are available and make decision about which to do within the constraints of available time, available budget and attractiveness of the activity. Once selected, any costs in doing the chosen activity are subtracted from the travel groups budget and any distance covered to reach the activity are recorded. When the travel group is ready to move to its next destination, the shortest route between origin and destination is determined based on the road network which has been added to the model at startup as a line shapefile. Agent movement is based on Nick Malleson's RepastCity model (Malleson, 2011). The time it takes to travel between nodes is determined by the distance divided by an average highway velocity and may be broken at somewhat regular intervals for such purposes as eating (at set times of the day), personal needs, or by the necessity of refueling. Later versions of the model will allow travel groups to make decision about whether or not to stop at an intervening node if it provides enough attraction. All agents maintain a record of distances travelled (to determine their contribution to greenhouse gases, i.e. their environmental yield), their total expenditure, i.e. financial yield, and activities or attractions undertaken at each node. At the end of its trip, the travel group is removed and a report is generated to compare against the IVS for the simulated period as a form of model validation. Once validated, the model can be used to 
evaluate different scenarios for the mix of tourists, or exogenous effects such as currency or petrol price fluctuations or the impacts of natural disasters.

\subsection{Incorporating survey data}

In developing the agent-based model for this project, a significant challenge has been in translating the interpretations of the interview data into an appropriate framework for driving agent decision-making behaviour and their interactions with other agents as well as their environment. The interview data informed model development in two primary ways: identification of the tourist agents and codification of the rules that guide their behaviours.

Identifying the objects has proven a challenge as there is a multitude of logical sets that could be used. We were ultimately guided by a hierarchical (or cascading) approach to the outcomes of the interview data to derive those objects. Since much of the analysis of tourism in New Zealand is broken down by nationality, the initial level of grouping attaches a nationality to each agent (a travel group). At a later stage, this also allows us to attach rough indications of available budget and daily spending habits (derived from the IVS). In addition, nationality allows us to predict the impact of culture on behavior (Hofstede, 2001). The initial version of the model simulates the period of 1 November 2008 to 28 February 2009 with inputs based on data from the IVS. This also allows us to validate model outputs against real data. Given the importance of Trip Type identified from the interviews, agents were initially grouped into Holiday, VFR Couple or VFR Family, Working Holiday or RTW classes with the distribution based on IVS data for the simulated period. The recorded length of stay "book ends" the trip and enables us to group the agents by the type of itinerary, being one of loop, triangle or stationary. Finally, attributes of each travel group are created for transport type and preferred level of accommodation, number of people in travel group, etc. The interviews also showed that tourists' decision-making behaviours evolved over the course of their stay, being broken into distinct thirds. The factors were all necessary to incorporate when formulating the rules that governed agent behavior. Given the complexities of the agents, rules governing choices made by travel groups are variations on a "take-the-best" heuristic. In later versions we will experiment with other versions to simulate more sophisticated processes, such as those outlined by Gigerenzer and Todd (1999). At the time of writing the model is still in the development stage and significant results will be presented when available.

\section{DISCUSSION AND CONCLUSIONS}

At this point in model development, there remain several key components of the problem that require further development. Most importantly, agents need the ability to determine their own itineraries, based on constraints of overall trip duration and time required to travel between nodes. Some of our recent field work indicates that for many tourists, their itineraries are relatively fixed by the time they've arrived in New Zealand and begun travelling. Nonetheless, there are certainly cases where travel groups decide their next destination on a day to day basis. In addition, there is the possibility that groups may decide upon a new destination while in transit because of unanticipated time demands, new information (perhaps gained during a refreshment stop), or rerouting due to environmental impacts (e.g. flooding, landslips). In the case of destination decisions, agents would need to be able to compare nodes based on some internal preferences and make a selection, incorporating other factors such as the travel time (which may be affected by group composition, such as the presence of young children) and the impact of travelling to a given node on the overall time available and the tradeoffs that may entail. In New Zealand's case, this is somewhat constrained by geography, especially in the South Island, where alternative destinations are generally in the same direction of travel.

Another key development will be an interface that makes it easier for laypeople to interact with the model and create their own scenarios. Discussions with the end users of the model will assist us with creating useful and informative visualizations of model outputs that move beyond the movement of travel groups between nodes.

Thus far, this project has posed some significant challenges in formulating the model, not least of which is the need to translate discursive accounts from tourists (in interviews) into executable heuristics that guide agent behaviors. This has required an interdisciplinary approach between social scientists, programmers, and economists. While challenging, we do feel that this has allowed us to develop a model grounded in the context of actual decisions made by tourists. This is essential if the model is to prove useful to government and industry decision makers in scenario planning. This project has focused on what it generally thought of as an area of unconstrained or free and open decision making, a situation that can make ABM approaches highly complicated and demanding conceptually. Our fieldwork has shown that there is a degree of 
Doscher et al., An agent-based model of tourist movements in New Zealand: implications for spatial yield

predictability in tourists' decision making that can help to constrain the modeling efforts, yet still leave room for unanticipated patterns to develop. We are hopeful that these efforts also support the extension of the ABM framework further into human decision contexts.

\section{REFERENCES}

Gigerenzer, G., and P.M. Todd (1999). Fast and frugal heuristics: the adaptive toolbox. In G. Gigerenzer, P. M. Todd, \& the ABC Research Group (Eds.), Simple Heuristics That Make Us Smart: 3-34. New York: Oxford University Press.

Hofstede, G. (2001). Culture's Consequences, Comparing Values, Behaviors, Institutions, and Organizations Across Nations. Thousand Oaks CA: Sage Publications.

Malleson, N. (2011). RepastCity - a demo virtual city. http://portal.ncess.ac.uk/wiki/site/mass/repastcity.html

Ministry of Economic Development (2011a). Key tourism statistics. http://www.tourismresearch.govt.nz/Documents/Key\%20Statistics/KeyTourismStatistics2011(July).p df

Ministry of Economic Development (2011b). International visitor survey. http://www.tourismresearch.govt.nz/Data--Analysis/International-tourism/International-Visitors/

North, M., N. Collier, and J. Vos (2006). Experiences in creating three implementations of the repast agent modeling toolkit. ACM Trans. Model Comp Simul 16(1):1-25

ROAD (Repast Organistaion for Architecture and Design) (2009). Repast home page: http://repast.sourceforge.net 